\title{
Segmentation of Remotely Sensed Images using Unsupervised 'Sampling- Resampling' based on Hopfield Type Neural Network
}

\author{
R. Cheryal Percy
}

Assistant Professor,

Department of Computer Applications,

Valliammal College for Women , Chennai - 600102

E-mail Id:cheryalpercy@gmail.com

\begin{abstract}
-
In this paper we propose a technique for performing unsupervised segmentation for satellite images using a 'sampling - resampling' based on Hopfield type Neural Network. The multi band values of the satellite images are grouped into clusters that are modeled using Gaussians. The parameters of Gaussian mixture models are learnt using Hopfield Type Neural Network. The purpose of this work is to show the effectiveness of the results obtained by using Hopfield type Neural Network rather than Bayesian parameter estimation. Each spatial position in the considered image is represented by neuron that is connected only to its neighboring units. It can be observed that the proposed technique have a better correspondence to the actual land features in the satellite images than compared with the results obtained by using the clustering technique like K-means Algorithm. The unsupervised techniques learns the class parameter by exploiting the structure of the unlabeled data .However, the numerical integration technique that are required for implementing Bayesian learning becomes complicated for practical applications, because of involving large data's than compared to the Hopfield type Neural Network model.
\end{abstract}

Index Terms: Bayesian, Remotesensing,K-means algorithm, modeling,multiband,Gaussians.

\section{Introduction}

The advancements in various remote sensing platforms have resulted in the production ofhuge amounts of satellite images. The Modern forms of satellite images are quite oftenmultispectral or hyper spectral, and provide more data in the spectral/frequency domainas well in the spatial domain; much more than what the human eye can 'see' from space andThere is a need for effective sorting, querying and browsing of information in these imagedatabases.Segmentation is a key step for performing higher level operations such as recognition,scene understanding, indexing etc. Applications ranges from areas related to agriculture,environment study, geophysical exploration to even national security and military relatedtasks. In all these domains, the quality of the result depends largely on the quality ofthe segmented satellite images.A various image segmentation techniques and algorithms have been proposed overthe years and [24] review existing techniques are specific to segmentation ofsatellite images. In general, segmentation approaches can be classified into edge or contourdetection based [24] [15], morphological region-based [22] and stochastic model basedapproaches It has been argued in [6] and [5], that a majority of techniquesbased on themorphological region growing or contour detection are not suitable for satelliteimage segmentation.

Supervised techniques requires some initial training data, based on which, the class parametersare learnt. But it is rather unrealistic for many practical applications where trainingdata may not be available. Unsupervised techniques learns the class parameters by exploitingthe structure of the (unlabeled) data.This paper presents a statistical modeling based segmentation technique where Hopfield Type Neural Network is used to update the parameters of the clusters formed by the pixels of the satellite image.However, numerical integration techniques 
that are required for implementing Hopfield Type Neural can become cumbersome for practical applications,particularly involving large data.To assess the effectiveness of the presented techniques, we consider a satellite image and compared the results provided by the proposed technique with the obtained data.This paper is organized as follows: Section II provides a brief description of the Hopfield neural network. Section III describes the Proposed Method of Images using Unsupervised 'SamplingResampling' based on Hopfield Type Neural Network. Section IV describe the Efficiency of Hopfield type Neural Network and Section V ,conclusions are drawn.

\section{Background : Hopfield Neural Networks}

The Hopfield neural network consists of set of neurons, the output of each neuron is fed back to each other units in the networks asillustrated in the Fig.1. It implies that there is no self-feedback and the synaptic weights are symmetric [18]. Hopfield defines the energy function of the networks by using architecture,i.e., the number of neurons, their output functions, threshold values, connections between the neurons and their strengths [19]. The energy function represents the complete status of the network.Hopfield shows that at each iterations the processing of the network, the energy value decreases and the network reaches a stable state when its energy value reaches the minimum value [13],[17].

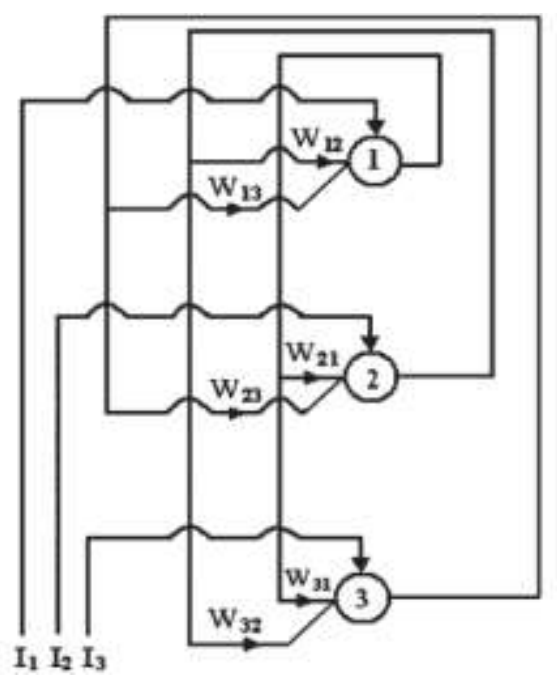

Fig 1. This is an example of a Hopfield network consistof three neurons.Neurons are represented by circles and connected lines between neurons show that the output of each neuron is fed back to other neurons.

The input data $U i$ to the generic $i$ th neuron comes from the two sources namely 1) input $\mathrm{V} j$ from other units and 2) external input bias Ii,which is a fixed bias applied externally to the unit $i$. thus the total input to the neuron I is given by

$$
U_{i}=\sum_{j=1, j \neq i}^{n} W_{i j} V_{j}+I_{i}
$$

where the weight Wijrepresents the synaptic interconnection from neuron $j$ to neuron $i$, and $n$ is the total number of units in the network. The connection strengths are assumed to be symmetric, i.e., Wij= Wji. The output Viof neuron $i$ is defined as;

$V i=g(U i)$

Where $\mathrm{g}($.$) is an activation function and there are$ two types of Hopfield (i.e., discrete

and continuous) based on the output values that a neuron can assume

\section{A.Discrete Model}

In the discrete model, the neurons are bipolar, i.e., the output $V i$ of neuron $i$ is either +1 or -1 . In this model, the activation function $g(\cdot)$ is defined according to the following thresholdfunction.

$$
V_{i}=g\left(U_{i}\right)= \begin{cases}+1, & \text { if } U_{i} \geq \theta_{i} \\ -1, & \text { if } U_{i}<\theta_{i}\end{cases}
$$

Where $\theta \mathrm{i}$ is the predefined threshhold of the neuron $i$. The energy function $\mathrm{E}$ of the discrete model is given by []

$$
E=-\sum_{i=1}^{n} \sum_{j=1, i \neq j}^{n} W_{i j} V_{i} V_{j}-\sum_{i=1}^{n} I_{i} V_{i}+\sum_{i=1}^{n} \theta_{i} V_{i} .
$$

\section{B. Continuous Model}

In this model, the output of a neuron is continuous and can assume any real value between 
$[-1,+1]$. In this continuous model, the activation function $g($.) must satisfies the following conditions: 1) it is a monotonic nondecreasing function and 2)g-1(.) exists.

A typical choice of the function(.) is

$$
g\left(U_{i}\right)=\frac{2}{1+e^{-\phi_{\mathbf{x}}\left(U_{i}-\tau\right)}}-1
$$

Where the parameter $\tau$ controls he shifting of the sigmoidal function $\mathrm{g}($.) along the abscissa , and the $\varphi i$ determines the steepness of the neuron $i$.the value of $\mathrm{G}(\mathrm{U} i)$ lies on $[-1,+1]$ and is equal to 0 at $\mathrm{U} i=\tau$. The energy function $\mathrm{E}$ of the continuous model.

\section{Image Change Detection Using 'Sampling- Resampling Based Hopfield Type Neural Network}

The satellite image consists of multivariate data points, or D-dimensional pixel values, where $\mathrm{D}$ is the number of bands or the channels in the satellite image. The pixel values are expected to form a certain number (say K) of cluster i.e. For example, pixels corresponding to water bodies concrete or built up regions, etc. These objectives of clustering pixel values involves

particular pixel value belongs to, at the same time learning certain class parameters.

\subsection{Preprocessing}

The physical features which are shown in a typical satellite image tend to be spatially clustered together. For example, the satellite image shown in Fig. 2, it is easy to identify regionswhere pixels corresponding to a particular land feature form spatial clusters. This strongspatial correlation between pixels corresponding to a particular class can be exploited to enhance the final segmented result.

Hence before clustering the pixels, a preprocessing step using the Bilateral filter

is performed on the satellite image. The Bilateralfilter is a non-linear filtration method thatsmoothness' a signal while preserving strong edges. And this has been used on a variety of imageprocessing and computer vision tasks

The traditional domain filteris applied to an image $f(r)$ produces an output image defined asfollows:

$$
\mathbf{h}(\mathbf{r})=k_{d}^{-1}(\mathbf{r}) \int_{-\infty}^{\infty} \int_{-\infty}^{\infty} f(\xi) c(\xi, \mathbf{r}) d \xi
$$

where $c(\xi \mathrm{r})$ measures the geometric closeness between the center pixel $r$ and a nearby

point $\xi . k d(r)$ is a normalizing function given by

$$
k_{d}(\mathbf{r})=\int_{-\infty}^{\infty} \int_{-\infty}^{\infty} c(\xi, \mathbf{r}) d \xi \text {. }
$$

\subsection{Segmentation}

Segmentation can be defined as partitioning an image into some non-overlapping meaningful homogeneous region, the term 'meaningful' is problem dependent. The success of an image analysis depends on the quality of segmentation. The main aim of segmentation is to reduce the information for simple analysis. Segmentation is also useful in Image Analysis and Image Compression.

Segmentation can be broadly classified as follows:

- Region Based

- $\quad$ Edge Based

- Threshold

- $\quad$ Feature Based Clustering

- Model Based

\section{a. Region Based}

In this technique the pixels that are related to an object are grouped for segmentation The thresholding technique is bound with region based segmentation. The area that is detected for segmentation should be closed. The Region based segmentation is also termed as "Similarity Based Segmentation" and there won't be any gap due to missing edge pixels in this region based segmentation.

The boundaries in the images are identified for segmentation and in each and every step at least one pixel is related to the region and is taken into consideration .After identifying the changes in the color and texture, the edge flow is converted into a vector. 


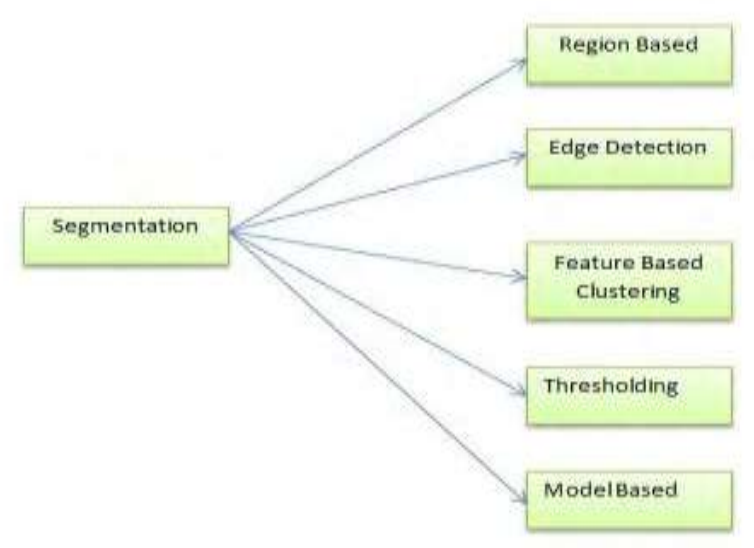

Fig.3 various types of segmentation

\section{b.Edge Based}

Segmentation can also be done by using edge detection techniques. In this technique the boundary is identified to segment. The Edges are detected to identify the discontinuities in the image. Edges corresponding to the region are traced by identifying the pixel value and it is compared with the values of the neighboring pixels. For this classification they use both fixed and adaptive feature of Support Vector Machine (SVM)

In this edge based segmentation there is no need for the detected edges to be closed.There are various edge detectors that are used to segment the image.

In that the well know is Canny edge detector and it has some step by step procedure for segmentation is mentioned in which is as follows:

1. To reduce the effect of noise, the surface of the image is smoothened by using Gaussian Convolution.

2. The Sobel operator is applied to the image to detect the edge strength and edge directions.

3. The edge directions are taken into considerations for non-maximal suppression i.e., the pixels that are not related to the edges are detected and are minimized.

4. Final step is for removing the broken edges in the image i.e., the threshold values of an image is calculated and then the pixel value is compared with the threshold value that is obtained. If the pixel value is higher than the threshold then, it is considered as an edge or else it is rejected.

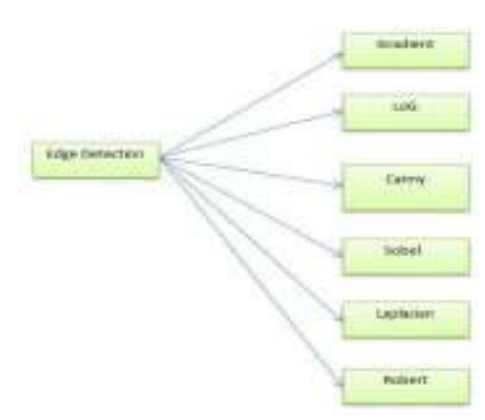

Fig 4.Types of Edge Detection

The Canny Edge Detection procedure is described in the flowchart
c.Threshold:

Thresholding is described as the easiest way of segmentation. It is done through the threshold values which are obtained from the histogram of those edges of the original image. The threshold values are obtained from the detected image. So, if the edge detections are accurate then the threshold too. Segmentations through thresholding has fewer computations compared to other techniques. Segmentation are based on "his ton". For a particular segment there may be set of pixels which is termed as "his ton". Roughness measure is followed by a thresholding method for image segmentation

Segmentation are done through adaptive thresholding. In the gray level points where the gradient is higher, is then added to thresholding surface for segmentation The drawback of this segmentation technique is that it is not suitable for complex images.

\section{d.Feature Based Clustering:}

Segmentation can also be done through Clustering. They follow a different procedure, where most of them apply the technique directly 
to the image but here the image is converted into histogram and then clustering is done on it. Pixel of the color image are clustered for segmentation using an unsupervised technique Fuzzy C. This procedure is applied for ordinary images. If it is a noisy image, it results to fragmentation.

The basic clustering algorithm i.e., K-means is used for segmentation in textured images. It clusters the related pixels to segment the image Segmentation is done through feature clustering and there it will be changed according to the color components. Segmentation purely depends on the characteristics of the image. Features are taken into account for segmentation. The Difference in the intensity and color values are used for segmentation .

For the segmentation of color image they use Fuzzy Clustering technique, which iteratively generates color clusters using Fuzzy membership function in color space regarding to image space. The technique is successful in identifying the color region . Real time clustering based segmentation. Virtual attention region is captured accurately for segmentation. Image is segmented coarsely by multithresholding .It is then refined by Fuzzy C-Means Clustering. The advantage are applied to any multispectral images

Segmentation approach for region growing is $\mathrm{K}$ Means Clustering. The Clustering technique for image segmentation is done with cylindrical decision elements of the color space. These surface is obtained through histogram and is detected as a cluster by thresholding. Seeded Growing Region (SRG) is used for segmentation. It has a drawback of pixel sorting for the labeling. So, to overcome this boundary oriented parallel pixel labeling technique is obtained to SRG .

\section{e.Model Based:}

The Markov Random Field (MRF) based segmentation is known as Model based segmentation. An inbuilt region smoothness constraint is presented in MRF which is used for color segmentation. The components of the color pixel tuples are considered as independent random variables for further processing. The MRF is combined with edge detection for identifying the edges accurately . there are correlations among the color components. Expectation-Maximization (EM) algorithm values the parameter is based on unsupervised operation. Multiresolution based segmented technique is named as "Narrow Band" and it is faster than the traditional approach. The initial segmentation is performed at coarse resolution and then at finer resolution. These process moves on in an iterative fashion. The resolution based segmentation is done only to the part of the image.

The segmentations may also be done by using Gaussian Markov Random Field (GMRF) where the spatial dependencies between pixels are considered for the process Gaussian Markov Model (GMM) based segmentation is used for region growing The extension of Gaussian Markov Model(GMM) detects the region as well as edge cues within the GMM framework.

\section{Efficiency of Hopfield Type Neural Network}

In this paper images of Mexico is chosen for an example ,here the optimal threshold value was computed by a manual trial and error procedure, i.e., generating the change detection by initializing the networks with different threshold values and computing the overall change detection error by using the available reference map.
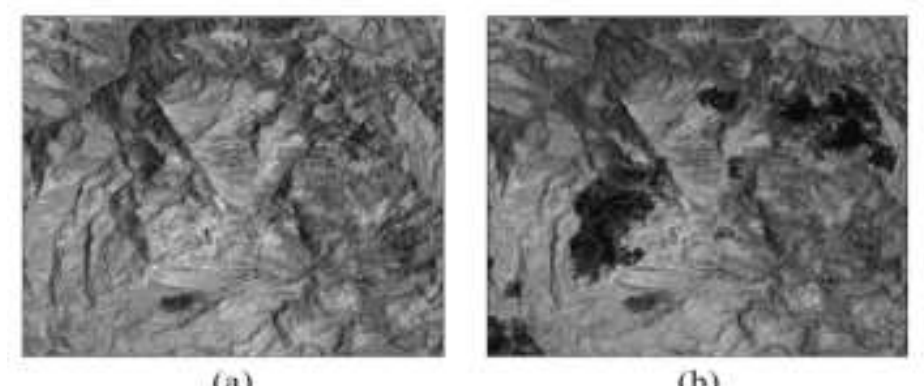

(b)

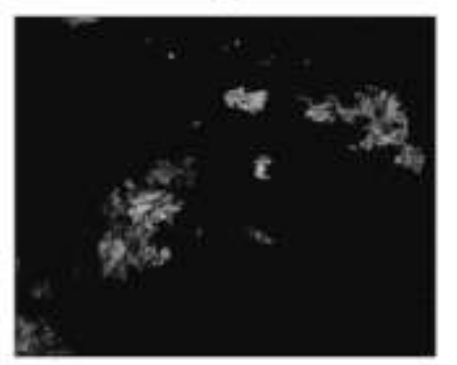

(c)

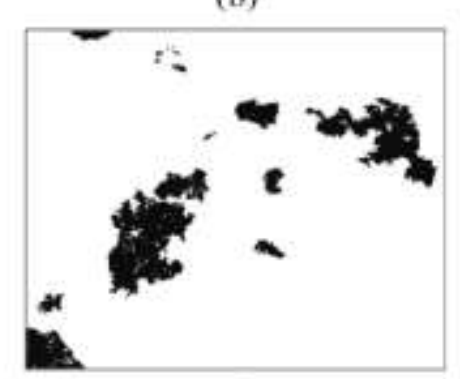

(d)

Fig.6. images of Mexico ,(a) Band 4 of the Landsat ETM+image acquired in April2000, (b) band 4of the Landsat ETM+image acquired in May 2002,(c) corresponding difference image generated by CVA technique, and (d) reference map of the changed area 
out between the optimal initialization threshold and the initialization threshold.

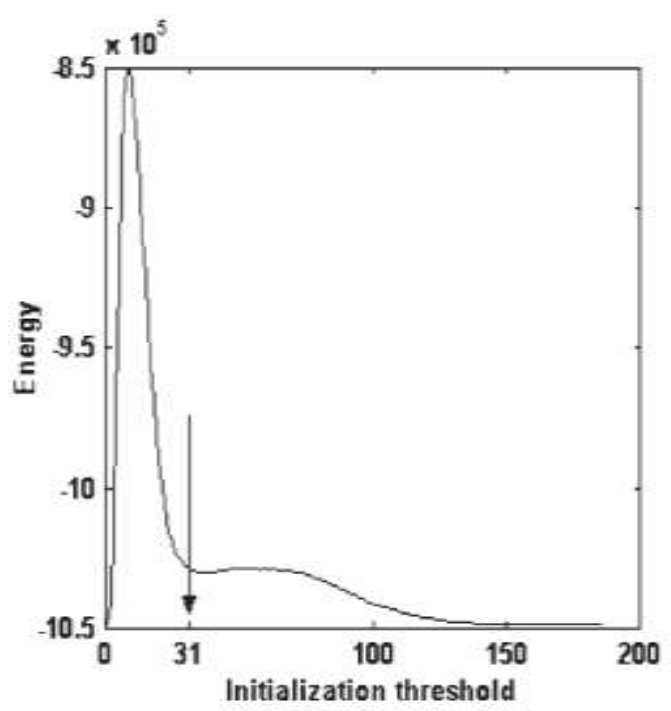

Fig.7. Behavior of the energy value versus the initialization values obtained by using Hopfield model.

\section{References}

[1] Singh, "Digital change detection techniques using remotely sensed data," Int. J. Remote Sens., vol. 10, no. 6, pp. 989-1003, 1989.

[2] J. A. Richards and X. Jia, Remote SensingDigital Image Analysis, 4th ed.Berlin, Germany: Springer-Verlag, 2006.

[3] J. Cihlar, T. J. Pultz, and A. L. Gray, "Change detection with synthetic aperture radar," Int. J. Remote Sens., vol. 13, no. 3, pp. 401-414, 1992.

[4] L. Bruzzone and S. B. Serpico, "An iterative technique for the detection of land-cover transitions in multitemporal remote-sensing images," IEEE Trans. Geosci. Remote Sens., vol. 35, no. 4, pp. 858-867, Jul. 1997.

[5] L. Bruzzone and D. Fernandez Prieto, “An adaptive parcel-based technique for unsupervised change detection," Int. J. Remote Sens., vol. 21, no. 4, pp. 817-822, 2000.

\section{Conclusion}

In this paper a comparative study of effectiveness of the Image segmentation is carried out and the experimental results obtained for the multitemporal dataset and the effectiveness of the Hopfield method is shown and we can also use this method for segmenting the remotely sensed images using Unsupervised 'sampling-resampling technique to obtain a effective results.

[6] S. Gopal and C. Woodcock, "Remote sensing of forest change using artificial neural networks," IEEE Trans. Geosci. Remote Sens., vol. 34, no. 2, pp. 398-404, Mar. 1996.

[7] T. Hame, I. Heiler, and J. S. MiguelAyanz, "An unsupervised change detection and recognition system for forestry," Int. J. Remote Sens., vol. 19, no. 6, pp. 10791099, 1998.

[8] [P. S. Chavez, Jr. and D. J. MacKinnon, "Automatic detection of vegetation changes in the southwestern United States using remotely sensed images," Photogramm.Eng. Remote Sens., vol. 60, no. 5, pp. 1285-1294, 1994.

[9] [K. R. Merril and L. Jiajun, "A comparison of four algorithms for change detection in an urban environment," Remote Sens. Environ., vol. 63, no. 2, pp. 95-100, 1998.

[10] [T. Fung, "An assessment of TM imagery for land-cover change detection," IEEE Trans. Geosci. Remote Sens., vol. 28, no. 4, pp. 681-684, Jul. 1990. 
[11] D. M. Muchoney and B. N. Haack, "Change detection for monitoring forest defoliation," Photogramm. Eng. Remote Sens., vol. 60, no. 10, pp. 1243-1251, 1994.

[12] L. Bruzzone and D. FernàndezPrieto, "Automatic analysis of the difference image for unsupervised change detection," IEEE Trans. Geosci. Remote Sens., vol. 38, no. 3, pp. 1171-1182, May 2000.

[13] J. R. G. Townshend and C. O. Justice, "Spatial variability of images and the monitoring of changes in the normalized difference vegetation index," Int. J. Remote Sens., vol. 16, no. 12, pp. 21872195, 1995.

[14] A. P. Dempster, N. M. Laird, and D. B. Rubin, "Maximum likelihood from incomplete data via the EM algorithm," J.R. Stat. Soc., vol. 39, no. 1, pp. 1-38, 1977.

[15] Y. Bazi, L. Bruzzone, and F. Melgani, “An unsupervised approach based on the generalized Gaussian model to automatic change detection in multitemporal SAR images," IEEE Trans. Geosci. Remote Sens., vol. 43, no. 4, pp. 874-887, Apr. 2005.

[16] [L. Bruzzone and D. FernàndezPrieto, “An adaptive semiparametric and context-based approach to unsupervised change detection in multitemporal remote-sensing images," IEEE Trans. Image Process., vol. 11, no. 4, pp. 452-466, Apr. 2002.

[17] [A. Ghosh, N. R. Pal, and S. K. Pal, "Object background classification using Hopfield type neural network," Int. J. Pattern Recognit. Artif.Intell., vol. 6, no. 5, pp. 989-1008, 1992.

[18] [S. Haykin, Neural Networks: A Comprehensive Foundation. Singapore: Pearson Education, 2003.Fourth Indian Reprint.
[19] [S. V. B. Aiyer, M. Niranjan, and F. Fallside, "A theoretical investigation into the performance of the Hopfield model," IEEE Trans. Neural Netw., vol. 1, no. 2, pp. 204-215, Jun. 1990.

[20] [J. J. Hopfield, "Neural networks and physical systems with emergent collective computational abilities," Proc. Nat. Acad. Sci., U.S.A., vol. 79,no. 8, pp. 2554-2558, Apr. 1982.

[21] Neurons with graded response have collective computational properties like those of two state neurons," Proc. Nat. Acad. Sci., U.S.A., vol. 81, no. 10, pp. 3088-3092, May 1984.

[22] S. K. Pal and D. DuttaMajumdar, Fuzzy Mathematical Approach to Pattern Recognition. New York: Halsted, 1986.

[23] A Rosenfeld and P. De La Torre, "Histogram concavity analysis as an aid in threshold selection," IEEE Trans. Syst., Man, Cybern., vol. SMC-13, no. 3, pp. 231-235, Mar. 1983. Press.

[24] N.R Pal and S.K Pal. A review on image segmentation techniques. Pattern Recognition, 26:1277\{1294, 1993.

[25] P.K Saha and J.K Udupa. Optimum image thresholding via class uncertainty and region homogeneity. IEEE Transactions on Pattern Analysis and Machine Intelligence, 23:689\{706, 2001. 